\title{
4-Hydroxymidazolam Measurement
}

National Cancer Institute

\section{Source}

National Cancer Institute. 4-Hydroxymidazolam Measurement. NCI Thesaurus. Code C154731.

The determination of the amount of 4-hydroxymidazolam present in a sample. 